Volume 8. No. 2, February 2020

International Journal of Emerging Trends in Engineering Research

Available Online at http://www.warse.org/IJETER/static/pdf/file/ijeter40822020.pdf

https://doi.org/10.30534/ijeter/2020/40822020

\title{
The Synthesis of the Optimal Decision Rule for Detecting an Object in a Joint Search and Detection of Objects by the Criterion of Maximum Likelihood
}

\author{
Hennadii Khudov $^{1}$, Andrii Zvonko ${ }^{2}$, Irina Khizhnyak ${ }^{3}$, Vasyl Shulezko ${ }^{4}$, Viacheslav Khlopiachyi ${ }^{5}$, \\ Viacheslav Chepurnyi ${ }^{6}$, Iryna Yuzova ${ }^{7}$ \\ ${ }^{1}$ Department of Radar Troops Tactic, Ivan Kozhedub Kharkiv National Air Force University, Kharkiv, Ukraine, \\ 2345kh_hg@ukr.net \\ ${ }^{2}$ Department of Rocket Artillery Armament, Hetman Petro Sahaidachnyi National Army Academy, Lviv, \\ Ukraine, zvonko2008@ukr.net \\ ${ }^{3}$ Department of Mathematical and Software Automated Control Systems, Ivan Kozhedub Kharkiv National Air \\ Force University, Kharkiv, Ukraine, khizh_ia@ukr.net \\ ${ }^{4}$ Department of Anti-aircraft Missiles Armament, Ivan Kozhedub Kharkiv National Air Force University, \\ Kharkiv, Ukraine, shulvas@ukr.net \\ ${ }^{5}$ Aviation Engineering Faculty, Ivan Kozhedub Kharkiv National Air Force University, Kharkiv, Ukraine, \\ 0674648530@ukr.net \\ ${ }^{6}$ Department of Tactic and Military Disciplines, Ivan Kozhedub Kharkiv National Air Force University, Kharkiv, \\ Ukraine, chvpximv@ukr.net \\ ${ }^{7}$ Department of Reserve Officers Training, Ivan Kozhedub Kharkiv National Air Force University, Kharkiv, \\ Ukraine,uzik25@ukr.net
}

\begin{abstract}
The weighted criterion of optimality of object detection during joint optimization of search and detection in the current area of view is specified taking into account the differential characteristics of the average risk. An expression is obtained for the likelihood ratio in the current area of view. An example of calculating the weight coefficient when making two-alternative decisions is presented.
\end{abstract}

Key words : Technical system, joint search and detection of objects, criterion of maximum likelihood, Bayes criterion of minimum average risk, two-alternative decisions.

\section{INTRODUCTION}

It is known [1-4] that when solving the task of joint search and detection of objects in technical systems, it is very important to synthesize the decisive rules for joint optimization of search and detection of objects. As an optimization criterion, as a rule, a Bayes criterion of minimum average risk is selected. Such a criterion is widely used in signal detection theory and has proven itself in practice.

To optimize the decision rule in detection systems, along with the Bayes criterion of minimum average risk, the maximum likelihood criterion is widely used $[2,5,6]$. Consider the features of using the maximum likelihood criterion for joint search and detection optimization in technical systems.

\subsection{Problem analysis}

In the problem of joint optimization of the search and detection of objects the ultimate is the task of detection [2, 7-10]. And the decision about object detection should be made based on the results of the analysis of signals that are received from the object of surveillance [2, 11-14].

The known results of solving optimization problems of object detection are reduced to the classical procedure for comparing the conditional likelihood ratio with a threshold [1-2, 15-16]. The achievements of the search theory are not taken into account. But a priori information about the location of the object in the search area is characterized by an integral indicator. The probability of the presence or absence of an object in the entire search area as a whole is considered as an integral indicator $[2,17]$.

Optimization methods, which are given in [2], consider search and detection as a single task only in the production plan. Solutions are obtained for the individual components of the task.

\section{MAIN MATERIAL}

A characteristic feature of solving the tasks of searching and detecting objects for a whole class of information systems is to take into account the probability of finding an object in the search area. 
With this in mind, the following expressions (1)-(3) can be written:

$$
\begin{gathered}
\int_{\Omega(t)} u(x) d x+\int_{\Omega(t)} \tilde{u}(x) d x=1 ; \\
\int_{\Omega(t)} u(x) d x \rightarrow P_{1}, \quad \text { for } \quad t \rightarrow T ; \\
\int_{\Omega(t)} \tilde{u}(x) d x \rightarrow 1-P_{1}, \quad \text { for } \quad t \rightarrow T ;
\end{gathered}
$$

where $u(x)$ - a priori probability density of the location of an object in a given search area $\Omega$ by space coordinates $x$;

$\tilde{u}(x)$ - a priori probability density of the absence of an object in a given search area $\Omega$ by space coordinates $x$;

$\Omega(t)$ - current area of view that meets the conditions $\Omega(t) \rightarrow \Omega$ for $t \rightarrow T$;

where $T$ - time of view of a given search area $\Omega$ (Figure 1 ); $P_{1}$ - a priori probability of the presence of an object in the current area of view $\Omega(t)$.

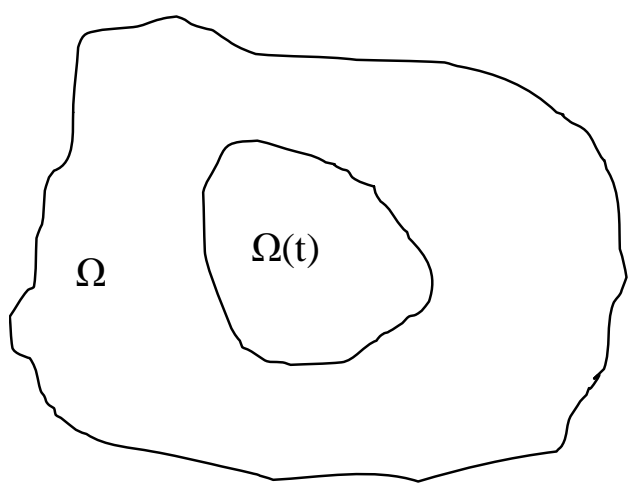

Figure 1: The ratio of the current viewing area $\Omega(t)$ and the search area $\Omega$

It is taken into account that the dimensions of the area of view are selectable so that the probability of the presence of an object of observation in it is equal to 1 .

Define the task of finding the optimal Bayes criterion for joint search and detection of objects in the current area of view $\Omega(t)$. It is necessary to consider the expressions (1)-(3).

As a criterion for the effectiveness of decision making choose the Bayes criterion for the minimum average risk. The average risk value can be found as (4):

$$
\begin{aligned}
& R(t)=I_{01} \int_{\Omega(t)} P\left(\gamma_{1} / H_{0}, x\right) \tilde{u}(x) d x+ \\
& +I_{10} \int_{\Omega(t)} P\left(\gamma_{0} / H_{1}, x\right) u(x) d x .
\end{aligned}
$$

To solve this task, the weight criterion is specified in the joint search and detection of objects in the current viewing area. And according to the selected criterion, two-alternative solutions are optimized.

Performing calculations taking into account expressions (1)-(3), obtain the following expression (4) for finding the optimal decision rule $\hat{A}(y)$. This expression (4) provides a minimum of average risk when deciding whether to detect an object in the current area of view $\Omega(t)$ of the search area $\Omega$ :

$$
\begin{aligned}
& \int_{Y} \int_{\Omega(t)} \hat{A}(y) W\left(y / H_{0}\right) \tilde{u}(x) \cdot \\
& \cdot\left(l(y, t) \frac{u(x)}{\tilde{u}(x)}-l_{0}\right) d x d y \Rightarrow \max ,
\end{aligned}
$$

where $l(y, t)=\frac{W\left(y / H_{1}\right)}{W\left(y / H_{0}\right)}-$ the conditional likelihood ratio, which characterizes the plausibility of hypotheses about the presence $H_{1}$ and absence $H_{0}$ of a signal from an object at the time $t$ when receiving an implementation $Y$;

$l_{0}=\frac{I_{01}}{I_{10}}-$ some weighting factor;

$I_{01}, I_{10}$ - elements of the loss matrix for incorrect decisions;

$\hat{A}(y)$ - functional of accepted implementation $y(t)$.

An additional factor $\frac{u(x)}{\tilde{u}(x)}$ was introduced in the expression (5). Denote it as $k_{a}$-weight of a priori data.

Analyze the expression (5). Since the probability density $W\left(y / H_{0}\right)$ and the probability density of the absence of an object in the area $\Omega(t)$ of the search area $\Omega \tilde{u}(x)$ are non-negative, the largest value of the weighted difference is achieved at the highest product values $\hat{A}(y)\left(l(y, t) \frac{u(x)}{\tilde{u}(x)}-l_{0}\right)$ for each possible value $y(t)$.

The values of the multiplications for possible values $\hat{A}(y)=1$ and $\hat{A}(y)=0$ are $l(y, t) \frac{u(x)}{\tilde{u}(x)}-l_{0}>0$ and 0 , respectively. 
Hennadii Khudov et al., International Journal of Emerging Trends in Engineering Research, 8(2), February 2020, 520 - 524

If $l(y, t) \frac{u(x)}{\tilde{u}(x)}>l_{0}$, then the value $l(y, t) \frac{u(x)}{\tilde{u}(x)}-l_{0}$ is large, which is achieved with solution $\hat{A}(y)=1$, which is preferable in this case.

If $l(y, t) \frac{u(x)}{\tilde{u}(x)}=l_{0}$, then the choice of solution $\hat{A}(y)$ is not real.

Then the optimality condition for a two-alternative hypothesis test in the current area $\Omega(t)$ of the search area $\Omega$ takes the following form (6):

$$
\hat{A}(y)=\left\{\begin{array}{lll}
1, & \text { if } & l(y, t) \frac{u(x)}{\tilde{u}(x)}>l_{0} ; \\
0, & \text { if } & l(y, t) \frac{u(x)}{\tilde{u}(x)}<l_{0} .
\end{array}\right.
$$

Thus, in order to make an optimal decision in the current area of view $\Omega(t)$ at the time $t$ after receiving the multi-dimensional implementation $Y$, it is necessary to calculate the conditional likelihood ratio $l(y, t)$ and the weighting factor $\frac{u(x)}{\tilde{u}(x)}$.

Weighted conditional likelihood ratio $l(y, t) \frac{u(x)}{\tilde{u}(x)}$ compared with threshold level $l_{0}$.

If weighted conditional likelihood ratio is below the threshold $l_{0}$, then hypothesis $H_{0}$ is accepted. If above the threshold $l_{0}$, then hypothesis $H_{1}$ is accepted.

Such a solution provides at time $t$ a minimum of average risk (4) and a maximum of a difference weight criterion (5).

Consider the weighting factor $\frac{u(x)}{\tilde{u}(x)}$ in more detail. From the expression (1) obtain the expression (7):

$$
\int_{\Omega(t)} \tilde{u}(x) d x=\int_{\Omega(t)}\left(\frac{1}{\int_{\Omega(t)} u(x) d x}-1\right) u(x) d x .
$$

Taking into account the need to fulfill (7) for any moment in time $0 \leq t \leq T$ and expressions (1) and (2), equate the integrands in (7):

$$
\tilde{u}(x)=u(x)\left(\frac{1}{\int_{\Omega(t)} u(x) d x}-1\right) .
$$

From the expression (8) have the expression (9):

$$
\frac{u(x)}{\tilde{u}(x)}=\frac{\int_{\Omega(t)} u(x) d x}{1-\int_{\Omega(t)} u(x) d x} .
$$

Introduce the notation $P(\Omega(t))=\int u(x) d x-$ the probability of finding an object in the current viewing area $\Omega(t)$ at a time $t$.

From expression (1) taking into account expressions (2) and (3), obtain the following expression for the weight factor (10):

$$
k_{a}=\frac{u(x)}{\tilde{u}(x)}=\frac{P(\Omega(t))}{1-P(\Omega(t))} .
$$

Taking into account (10) the expression of the optimization condition for a two-alternative hypothesis test in the current search area $\Omega(t)$ of the search area $\Omega$ takes the following expression (11):

$$
\hat{A}(y)=\left\{\begin{array}{lll}
1, & \text { if } & \frac{P(\Omega(t))}{1-P(\Omega(t))}>l_{0} ; \\
0, & \text { if } & \frac{P(\Omega(t))}{1-P(\Omega(t))}<l_{0} .
\end{array}\right.
$$

Thus, in order to make an optimal decision in the current area of view $\Omega(t)$ at a time $t$ after receiving a multidimensional implementation $Y$, the conditional likelihood ratio $l(y, t)$ and weighting factor $\frac{P(\Omega(t))}{1-P(\Omega(t))}$ should be calculated.

Weighted conditional likelihood ratio $l(y, t) \frac{P(\Omega(t))}{1-P(\Omega(t))}$ compared with threshold level $l_{0}$. If it is below the threshold, then hypothesis $H_{0}$ is accepted; otherwise, hypothesis $H_{1}$ is accepted. Such a solution provides at time $t$ a minimum of average risk (4) and a maximum of a difference weight criterion (5).

As an example, consider the dependence of the weight factor (10) on probability $P(\Omega(t))$. A graph of this dependence is presented in the Figures 1-3. 
Figure 2 shows the dependence of the weight of a priori data on the probability of finding an object in the current area of view $P(\Omega(t))$.

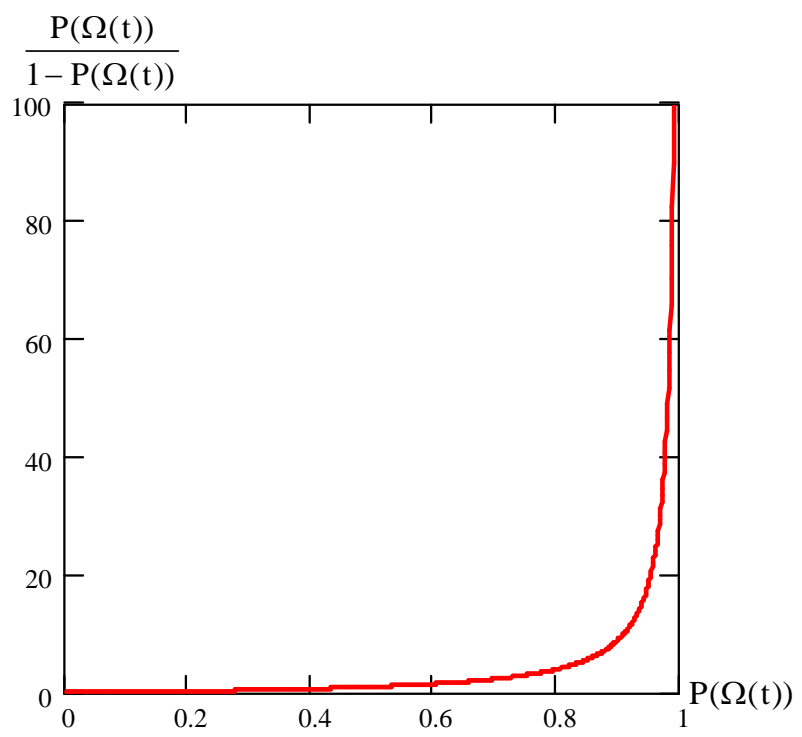

Figure 2: Dependence of the weight of a priori data on the value $P(\Omega(t))$

The graph shows a sharp increase in the weight of a priori data when the probability $P(\Omega(t))$ approaches one.

Figures 3-4 show a more detailed dependence of the weight of a priori data within different limits of the numerical range of probability changes $P(\Omega(t))$.

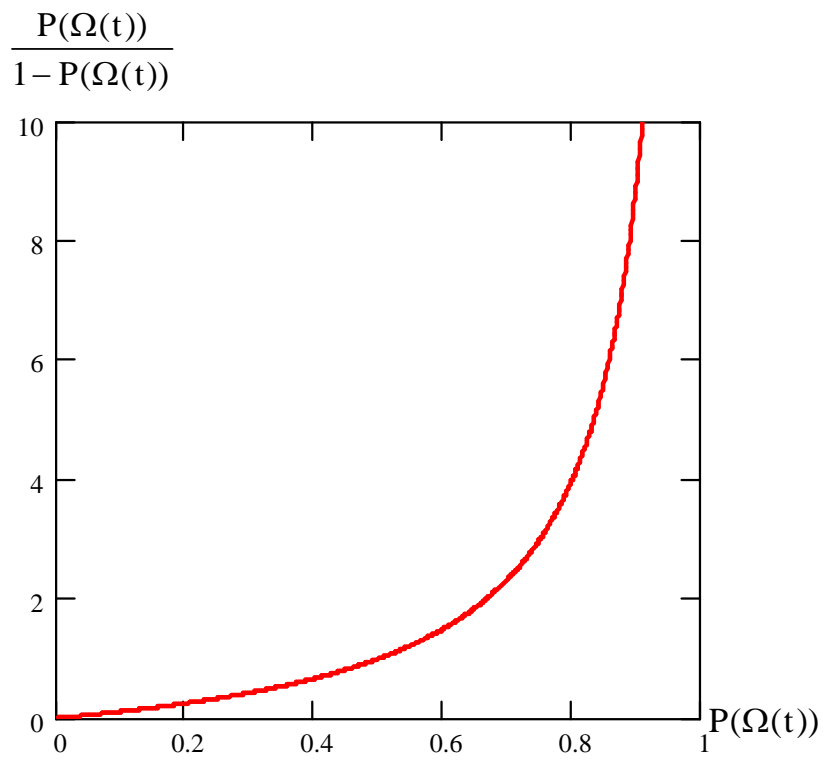

Figure 3: Dependence of the weight of a priori data on the value $P(\Omega(t))$

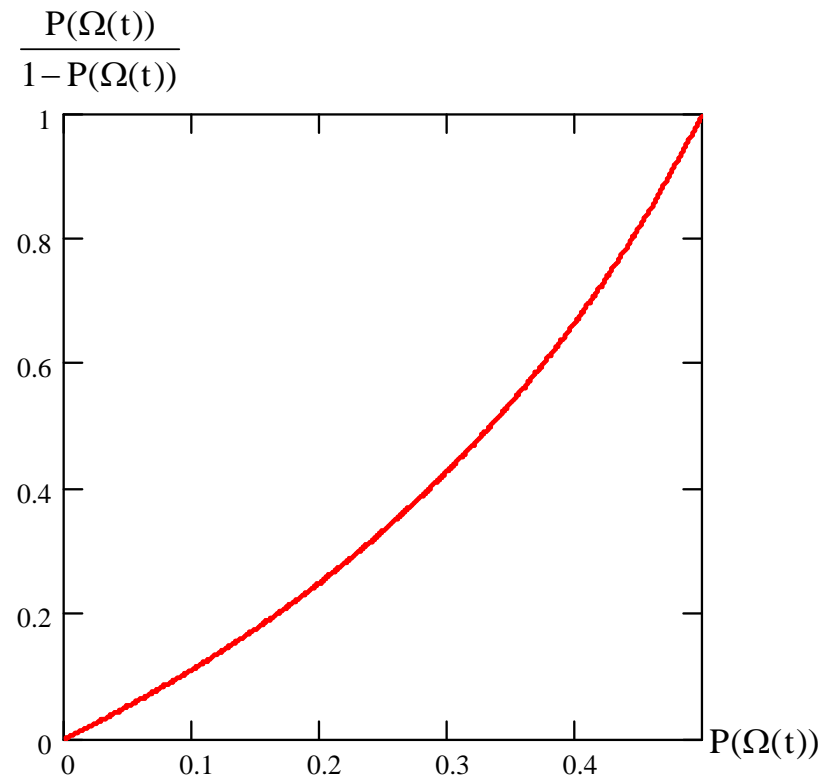

Figure 4: Dependence of the weight of a priori data on the value $P(\Omega(t))$

A characteristic feature of the weight of a priori data:

at a priori probability $P(\Omega(t)) \rightarrow 1$ weight of a priori data $k_{a}=\frac{P(\Omega(t))}{1-P(\Omega(t))} \rightarrow \infty$;

at $P(\Omega(t)) \rightarrow 0-k_{a}=1$.

When $P(\Omega(t))=0,5$ and $k_{a}=1$, the decisive rule in the joint optimization of the search and detection goes into the well-known rule in detection objects.

\section{CONCLUSION}

Thus, the weight criterion is clarified in the joint search and detection of objects in the current area of view. Optimized two-alternative solutions according to this criterion. Synthesis of the decision rule was carried out during a two-alternative test of hypotheses in the current area and joint optimization of the search and detection of objects by the criterion of maximum likelihood.

In further research, it is necessary:

to obtain expressions for the object search strategy;

to define a class of search strategies;

to develop practical recommendations for calculating the weight coefficient, depending on the nature of the tasks to be solved and the features of technical systems for various purposes. 


\section{REFERENCES}

1. R. B. Girshick, J. Donahue, T. Darrell, and J. Malik. Rich feature hierarchies for accurate object detection and semantic segmentation, in IEEE CVPR, 2014, pp. 580-587.

2. H. Khudov, I. Khizhnyak, F. Zots, G. Misiyuk, and O. Serdiuk. The Bayes Rule of Decision Making in Joint Optimization of Search and Detection of Objects in Technical Systems, IJETER, № 8(1), 2020, pp. 7-12. DOI: https://doi.org/10.30534/ijeter/2020/02812020.

3. I. Ruban, H. Khudov, O. Makoveichuk, I. Khizhnyak, V. Khudov, V. Podlipaiev, V. Shumeiko, O. Atrasevych, A. Nikitin, and R. Khudov. Segmentation of opticalelectronic images from on-board systems of remote sensing of the Earth by the artificial bee colony method, Eastern-European Journal of Enterprise Technologies, № 2/9 (98), 2019, pp. 37-45. DOI: https://doi.org/10.15587/1729-4061.2019.161860.

4. M. Iasechko, M. Mozhaiev, I. Manzhai, M. Volk, V. Manoylo, O. Ochkurenko, D. Maksuita, V. Larin, A. Markov, and O. Kostyria. Conditions for Reliable Transmission of Information Over Long Distances Using a Powerful Electromagnetic Radiation, IJATCSE. $\quad 8(1), \quad 2020, \quad$ pp. 138-144. DOI: https://doi.org/10.30534/ijeter/2020/18812020.

5. V. Lishchenko, V. Chaliy, G. Khudov, and A. Zvonko. Proposals for Improving of Air Surveillance Informativity in MIMO Radar Systems Based on Two-Dimensional Radars, in Intern. Scient.-Pract. Conf. Problems of Infocommunications. Science and Technology (PIC S\&T), 2018, pp. 153-156. DOI: https://doi.org/10.1109/infocommst.2018.8632052.

6. H. Khudov, A. Fedorov, D. Holovniak, and G. Misiyuk. Improving the Efficiency of Radar Control of Airspace with the Multilateration System Use, in Intern. Scient.-Pract. Conf. Problems of Infocommunications. Science and Technology (PIC S\&T), 2018, pp. 680-684. DOI: https://doi.org/10.1109/infocommst.2018.8632141.

7. H. Khudov, I. Ruban, O. Makoveichuk, H. Pevtsov, V. Khudov, I. Khizhnyak, S. Fryz, V. Podlipaiev, Y. Polonskyi, and R. Khudov. Development of methods for determining the contours of objects for a complex structured color image based on the ant colony optimization algorithm, Eureka: Physics and Engineering, № 1, 2020, pp. 34-47. DOI: https://doi.org/10.21303/2461-4262.2020.001108.

8. V. Lishchenko, H. Khudov, V. Tiutiunnyk, V._Kuprii, F._Zots, and G._Misiyuk. The Method of Increasing the Detection Range of Unmanned Aerial Vehicles In Multiradar Systems Based on Surveillance Radars, in 2019 IEEE 39th International Conference on Electronics and Nanotechnology (ELNANO), 2019. DOI: https://doi.org/10.1109/ELNANO.2019.8783263.

9. O. Turinskyi, M. Iasechko, V. Larin, D. Dulenko, V. Kravchenko, O. Golubenko, D.Sorokin, and
O. Zolotuin. Model and development of plasma technology for the protection of radio-electronic means of laser emission, IJATCSE. 8(5), 2019, pp. 2429-2433. doi:10.30534/IJATCSE/2019/85852019.

10. H. Khudov, A. Zvonko, S. Kovalevskyi, V. Lishchenko, F. Zots. Method for the detection of smallsized air objects by observational radars, Eastern-European Journal of Enterprise Technologies, № 2/9 (92), 2018, pp. 61-68.

DOI:https://doi.org/10.15587/1729-4061.2018.126509.

11. I. Ruban, V. Khudov, O. Makoveichuk, H. Khudov, and I. Khizhnyak. A Swarm Method for Segmentation of Images Obtained from On-Board Optoelectronic Surveillance Systems, in Intern. Scient.-Pract. Conf. Problems of Infocommunications. Science and Technology (PIC S\&T), 2018, pp. 613-618. DOI: https://doi.org/10.1109/infocommst.2018.8632045.

12. I. Ruban, H. Khudov, V. Khudov, I. Khizhnyak, and O. Makoveichuk. Segmentation of the images obtained from onboard optoelectronic surveillance systems by the evolutionary method, Eastern-European Journal of Enterprise Technologies, № 5/9 (89), 2017, pp. 49-57. DOI: https://doi.org/10.15587/1729-4061.2017.109904.

13. I. Ruban, H. Khudov, O. Makoveichuk, M. Chomik, V. Khudov, I. Khizhnyak, V. Podlipaiev, Y. Sheviakov, O. Baranik, and A. Irkha. Construction of methods for determining the contours of objects on tonal aerospace images based on the ant algorithms, Eastern-European Journal of Enterprise Technologies, № 5/9 (101), 2019, pp. 25-34. https://doi.org/10.15587/1729-4061.2019.177817.

14. Ruban I., Khudov H., Makoveichuk O., Khizhnyak I., Lukova-Chuiko N., Pevtsov G., Sheviakov Y., Yuzova I., Drob Y., and Tytarenko O. Method for determining elements of urban infrastructure objects based on the results from air monitoring, Eastern-European Journal of Enterprise Technologies, № 4/9 (100), 2019, pp. 52-61.

DOI: https://doi.org/10.15587/1729-4061.2019.174576.

15. I. Ruban, V. Khudov, H. Khudov, and I. Khizhnyak. An Improved Method for Segmentation of a Multiscale Sequence of Optoelectronic Images, in Intern. Scient.-Pract. Conf. Problems of Infocommunications. Science and Technology (PIC S\&T), 2017, pp. 137-140. DOI: https://doi.org/10.1109/INFOCOMMST.2017.8246 367.

16. T. D. Wickens. Elementary signal detection theory, New York, NY: Oxford University Press, 2002, 277 p.

17. M. Iasechko, Y. Gnusov， I. Manzhai， O. Uhrovetskyi, V. Manoylo, A. Iesipov, O. Zaitsev, M. Volk, and O. Volk. Determination of Requirements for the Protection of Radio-Electronic Equipment from the Terroristic Influence by Electromagnetic Radiation, IJATCSE. 7(12), 2019, pp. 772-777. DOI: https://doi.org/10.30534/ijeter/2019/077122019. 